\title{
Myeloproliferative Neoplasms
}

\author{
Nicolaus Kröger and Yves Chalandon
}

\subsection{Primary and Post ET/PV Myelofibrosis}

Nicolaus Kröger

\subsubsection{Definition and Risk Scores}

Polycythemia vera (PV) and essential thrombocythemia (ET) have a favorable outcome without need for allo-HSCT unless the disease progressed to post-ET/PV myelofibrosis or secondary AML (Lussana et al. 2014).

Primary myelofibrosis (PMF) or post-ET/PV myelofibrosis is one of the Philadelphia-negative myeloproliferative neoplasms (MPN) with worst survival which is approximately 6 years. AlloHSCT can cure a substantial number of patients but is still not universally applicable due to toxicity which leads to therapy-related morbidity and mortality.

\footnotetext{
N. Kröger $(\bowtie)$

Department of Stem Cell Transplantation University Medical Center Hamburg-Eppendorf Hamburg Germany e-mail: nkroeger@uke.de

Y. Chalandon $(\bowtie)$

Department of Oncology, Hematology Division Hôpitaux Universitaires de Genève,

University of Geneva

Geneva Switzerland

e-mail: yves.chalandon@hcuge.ch
}

\subsubsection{Transplant Results in Myelofibrosis}

In the late 1980s and the early 1990s, the feasibility of allo-HSCT for myelofibrosis could be shown in small reports. One multicenter report described in a retrospective study with MAC in relatively young patients (median age 42 years) with a NRM of $27 \%$ and a 9\% incidence of graft failure. The OS and PFS was $47 \%$ and $39 \%$ at 5 years (Guardiola et al. 1999). A single-center study from Seattle included 104 patients most of whom received allo-HSCT after MAC, and NRM at 5 years of $34 \%$ and OS at 7 years of $61 \%$ were reported (Deeg et al. 2003).

The evidence of graft-versus-myelofibrosis effect was documented by responses to DLI after failure of allo-HSCT (Byrne et al. 2000). RIC for myelofibrosis was investigated in two prospective studies. The EBMT published results of 103 patients who received a BU/FLU-based RIC followed by related or unrelated HSCT. The median age was 55 years, and the NRM at 1 year was $16 \%$. Cumulative incidence of relapse was $22 \%$ at 3 years. PFS and OS at 5 years were $51 \%$ and $67 \%$, respectively. Advanced age and HLAmismatched donor were independent predictive factors for reduced survival (Kroger et al. 2009). A recent update of the study after a median follow-up of 60 months showed an 8-year OS of $65 \%$ with stable plateau. Five-year DFS was $40 \%$, and 5-year cumulative incidence of relapse/ progression was $28 \%$ with 3-year NRM of $21 \%$.

The Myeloproliferative Disorders Research Consortium performed also a prospective phase II trial including 66 patients with primary or postET/PV myelofibrosis investigating a reduced 
conditioning regimen with MEL/FLU. With a median follow-up of 25 months, OS was $75 \%$ in the sibling group and only $32 \%$ in the unrelated group due to a higher NRM in the URD group (59\% vs. 22\%) (Rondelli et al. 2014). Other studies using RIC or MAC confirmed the curative effect of allo-HSCT irrespectively of the intensity of the conditioning (summarized in Kröger et al. 2015a).

\subsubsection{Disease-Specific Risk Factors}

Patients with PMF or post-ET/PV myelofibrosis have a median survival of approximately 6 years, but survival varies from less than 2 to more than 15 years. Risk scores (see Table 76.1) such as IPSS (Cervantes et al. 2009), dynamic IPSS (DIPSS) (Passamonti et al. 2010), or DIPSS plus (Gangat et al. 2011) are currently used in clinical practice to determine the prognosis of patients with PMF. More recently molecular markers have been introduced into the PMF risk score, (Guglielmelli et al. 2018) and a specific score for post-ET/PV myelofibrosis has been proposed (Passamontiet al. 2017; Kroger et al. 2015a). The EBMT/ELN consensus paper recommended allo-HSCT for patients less than 70 years with an estimated median survival of less than 5 years. This would include patients with IPSS or DIPSS intermediate- 2 and high risk and is based on a comparison between transplanted and non-transplanted patients in the pre-ruxolitinib era (Kroger et al. 2015a, b). Patients with intermediate-1 risk can be considered for allo-HSCT if other high-risk features such as ASXL1 mutation, more than $2 \%$ peripheral blasts, refractory transfusion-dependent anemia, or adverse cytogenetics according to DIPSS plus are present (Kroger et al. 2015a).

\subsubsection{Transplant-Specific Risk Factors}

In most of the transplant studies, alternative donors were associated with a worse outcome independent of disease-specific risk factors. CBT resulted in a high risk of graft failure (Robin et al. 2014). Haplo-identical donor with PT-CY as GVHD prophylaxis is currently under investigation, but more recent EBMT data reported a 5-year survival of only 38\% (Raj et al. 2016).

The intensity of the conditioning regimen has not been investigated within prospective studies, but retrospective comparisons of MAC and RIC preparative regimens resulted in similar outcome. Because of the reduced toxicity and a generally older age of patients with myelofibrosis, RIC regimens are currently used more frequently and account for about two-thirds of allotransplants for myelofibrosis reported to the EBMT registry.

Table 76.1 Prognosis risk scores for myelofibrosis

\begin{tabular}{|c|c|c|}
\hline Score & Adverse factors (puntos) & Risk group and median SRV \\
\hline IPSS & $\begin{array}{l}\text { Age }>65 \text { years }(1 \mathrm{p}) \\
\text { Constitutional symptoms }(1 \mathrm{p}) \\
\mathrm{Hb}<100 \mathrm{~g} / \mathrm{L}(1 \mathrm{p}) \\
\text { Leucocytes }>25 \times 10^{9} / \mathrm{L}(1 \mathrm{p}) \\
\text { Blasts in } \mathrm{PB} \geq 1 \%(1 \mathrm{p})\end{array}$ & $\begin{array}{l}\text { Low }(0 \mathrm{p}), 11.3 \text { years } \\
\text { Intermediate- } 1(1 \mathrm{p}), 7.9 \text { years } \\
\text { Intermediate- } 2(2 \mathrm{p}), 4 \text { years } \\
\text { High }(3-5 \mathrm{p}), 2.3 \text { years }\end{array}$ \\
\hline DIPSS & $\begin{array}{l}\text { Age }>65 \text { years }(1 \mathrm{p}) \\
\text { Constitutional symptoms }(1 \mathrm{p}) \\
\mathrm{Hb}<100 \mathrm{~g} / \mathrm{L}(2 \mathrm{p}) \\
\text { Leucocytes }>25 \times 10^{9} / \mathrm{L}(1 \mathrm{p}) \\
\text { Blasts in } \mathrm{PB} \geq 1 \%(1 \mathrm{p})\end{array}$ & $\begin{array}{l}\text { Low }(0 p) \text {, not reached } \\
\text { Intermediate- } 1(1-2 p):, 14.2 \text { years } \\
\text { Intermediate-2 }(3-4 p), 4 \text { years } \\
\text { High }(5-6 p), 1.5 \text { years }\end{array}$ \\
\hline DIPSS plus & 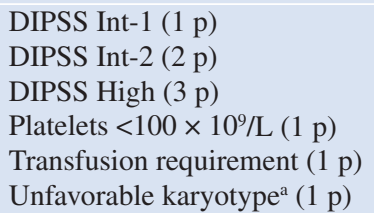 & $\begin{array}{l}\text { Low }(0 \mathrm{p}), 15.4 \text { years } \\
\text { Intermediate- } 1(1 \mathrm{p}), 6.5 \text { years } \\
\text { Intermediate- } 2(2-3 \mathrm{p}), 2.9 \text { years } \\
\text { High }(4-6 \mathrm{p}), 1.3 \text { years }\end{array}$ \\
\hline
\end{tabular}

DIPSS: https://qxmd.com/calculate/dipss-prognosis-in-myelofibrosis

DIPSS-plus: https://qxmd.com/calculate/dipss-plus-score-for-prognosis-in-myelofibrosis

${ }^{\mathrm{a}}+8 ;-7 / 7 \mathrm{q}-;-5 / 5 \mathrm{q}-;$ i17q; 12p-; rearrangement $11 \mathrm{q} 23$ 


\subsubsection{Patient-Specific Risk Factors}

Age is a significant patient-specific risk factor for outcome after allo-HSCT (Scott et al. 2012; Kroger et al. 2015a). Besides age, comorbidities and geriatric assessments (see Chap. 11) also impact on outcome after allo-HSCT but have not been studied especially in myelofibrosis patients to date.

\subsubsection{Role of Splenectomy and JAK Inhibition}

Splenomegaly is a hallmark of myelofibrosis and may have an impact on engraftment and graft function after HSCT. Splenectomy is an option to reduce spleen size prior to transplantation, but high morbidity and even mortality have been reported (Tefferi et al. 2000). Spleen irradiation to reduce spleen size has been reported successfully in single cases prior to conditioning. Since ruxolitinib is approved for myelofibrosis, the drug can be used prior to transplantation to improve constitutional symptoms and to reduce spleen size. The European LeukemiaNet and the
European Society for Blood and Marrow Transplantation recommend the use of ruxolitinib at least 2 months prior to HSCT and a careful weaning prior to conditioning to avoid the rebound phenomenon. More recent data suggest better outcome after HSCT if patients received transplant after responding to ruxolitinib rather than postponing the transplant until ruxolitinib failure (Shanavas et al. 2016).

\subsubsection{Impact of Molecular Remission}

About $90 \%$ of myelofibrosis patients harbor one of the driver mutations JAK2V617F, calreticulin (CALR), or MPL which are used to monitor MRD in PB by highly sensitive qPCR or digital PCR to determine molecular remission (Wolschke et al. 2017). In a retrospective single center experience, no achievement of molecular remission on day 180 post-allograft was associated with a significant higher incidence of a subsequent clinical relapse. Due to a graft-versus-myelofibrosis effect, donor lymphocyte infusion has been successfully applied in patients with residual to induce a molecular remission (Fig. 76.1).

\section{Managing stem cell tranplatation in myelofibrosis}

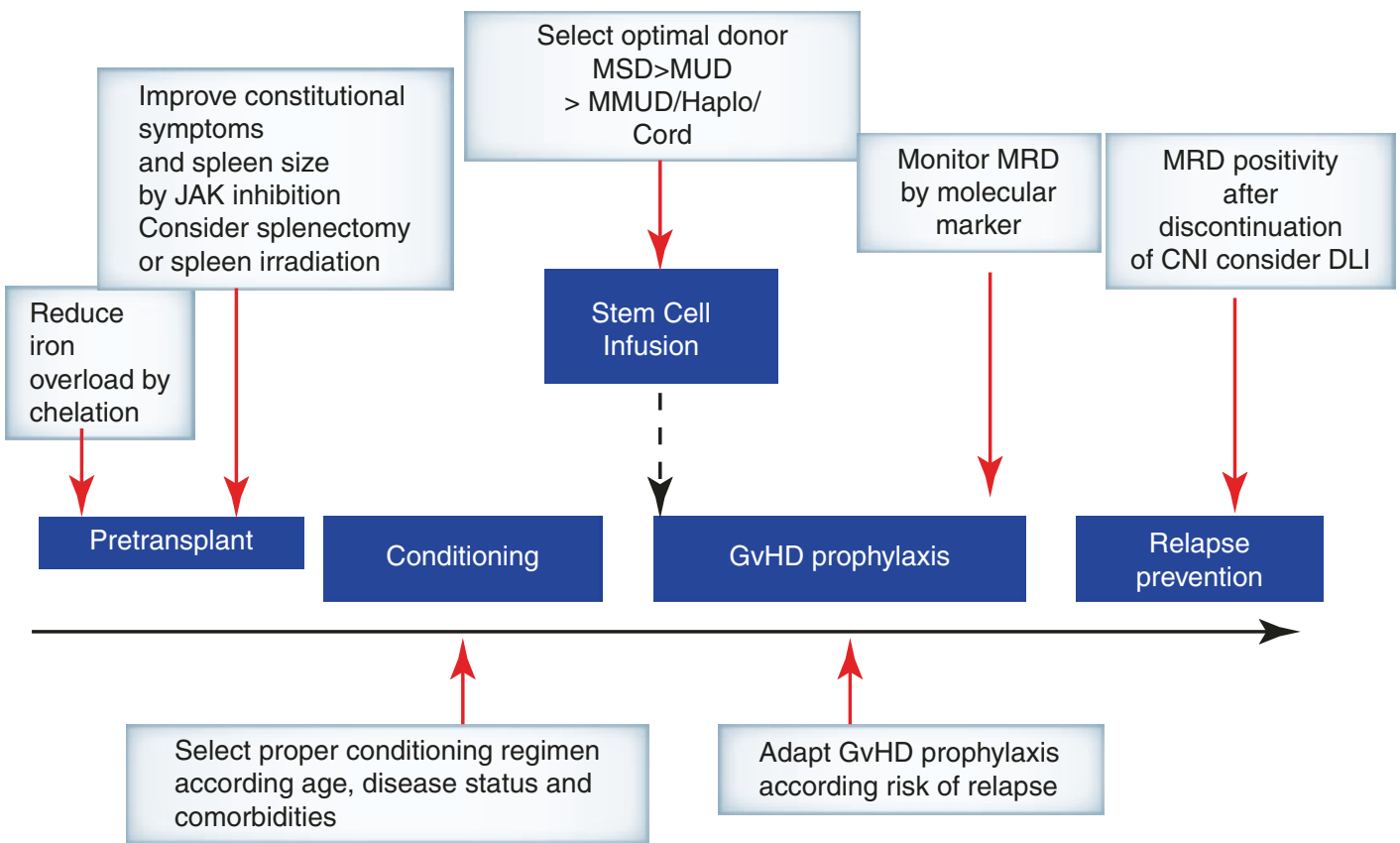

Fig. 76.1 $M S D$ matched sibling donor, $M U D$ matched unrelated donor, $M M U D$ mismatched unrelated donor, $M R D$ minimal residual disease, $C N I$ calcineurin inhibitor, GVHD graft-versus-host disease 
Furthermore, BM fibrosis is another hallmark of the disease, with rapid regression after allo-HSCT suggesting that fibrogenesis is a highly dynamic process (Thiele et al. 2005). Systematic investigations have shown that about $60 \%$ of the patients have a complete or nearly CR of BM fibrosis on day +100 , and the percentage of patients increased to $90 \%$ at day +180 . Notably, those patients with a rapid resolution of $\mathrm{BM}$ fibrosis had the best longterm outcome.

\section{Key Points}

- Primary or post-ET/PV myelofibrosis can only be cured by allo-HSCT which can induce molecular remission and resolution of bone marrow fibrosis.

- Indication for allo-HSCT is recommended for patients younger than 70 years and a median survival expectation of less than 5 years such as risk score intermediate or high risk according to DIPPS or intermediate-1 risk with additional risk factors.

- Splenectomy prior to transplant is not recommended, but patients with large spleen may benefit from JAK inhibitor treatment prior to transplantation.

- Major risk factors for worse outcome are advanced age and not fully HLAmatched donor.

\subsection{Chronic Myeloid Leukemia}

Yves Chalandon

\subsubsection{Definition, Epidemiology, Diagnosis, and Classification}

Chronic myeloid leukemia (CML) is a clonal myeloproliferative disorder of the HSC. CML was the first leukemia described and the first to be characterized by a consistent chromosomal aberration, the 22q- or "Philadelphia" $(\mathrm{Ph})$ chromosome, later identified as a reciprocal translocation, $t(9 ; 22)$, encoding the BCR-ABL oncoprotein.
CML is the most common of the myeloproliferative disorders. The incidence is $0.4-1.75$ per 100,000 population per year, and it increases with age (Höglund et al. 2015). The disease can occur at any age, but the median age at presentation ranges between 45 and 55 years. There is a slight male predominance, with a male to female ratio of 1.3:1.

CML present initially as an indolent or chronic phase (CP), easily controlled with treatment. The natural history continues with a bi- or triphasic stage, becoming more aggressive through accelerated phase (AP) and then blast crisis (BC) or directly from $\mathrm{CP}$ to $\mathrm{BC}$.

\subsubsection{Risk Factors and Prognostic Index}

Several multivariate-derived prognostic models and staging have been proposed to help define individual prognosis and allow assigning patients to different strategies of therapy based on risks. The most commonly used are the Sokal and Hasford one (Sokal et al. 1984; Hasford et al. 1998).

The benefit of allo-HSCT is that it can provide cure, but the clear disadvantage is its association with considerable morbidity and mortality, which typically occur early post procedure. Outcome can be improved by better selection of those most likely to benefit. In this context the EBMT developed a risk score for patients with CML, based on five variables: donor type, disease phase, recipient age, donor/recipient gender combination, and interval from diagnosis to transplant, which together results in a score of 0-7 (see risk factors in Chap. 11) (Gratwohl et al. 1998).

Results of transplant are now highly predictable based on these five factors. It is worth remembering that the EBMT or "Gratwohl" score was developed in the mid-1990s and was based on 3142 patients transplanted between 1989 and 1996 (Fig. 76.2a). With overall improvements in supportive care, it would be reasonable to expect that a similar analysis performed on patients transplanted more recently would demonstrate improved results across all-risk scores. However, the analysis is complicated by the change in approach to management of CML. During the period of the original analysis, allo-HSCT was the treatment of choice for all patients. Since 2000 

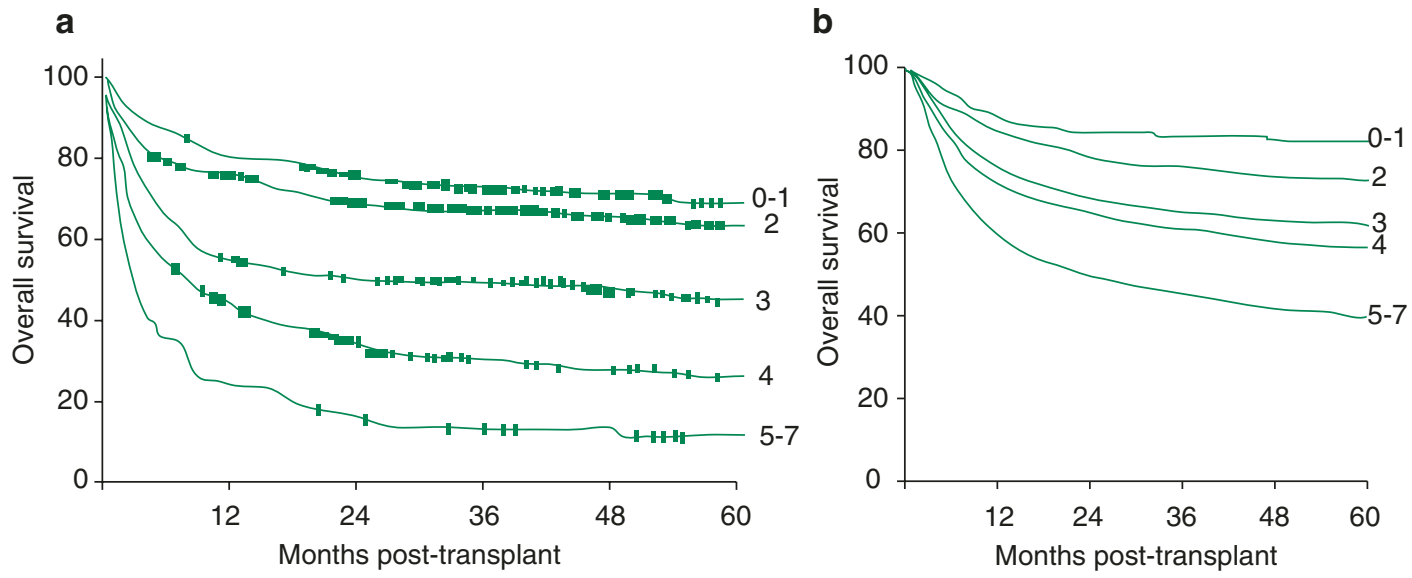

Fig. 76.2 (a) OS of CML patients after allo-HSCT according to EBMT risk score. Original curves published in 3142 patients transplanted between 1989 and 1996.

allo-HSCT has been replaced by tyrosine kinase inhibitors (TKI) as frontline therapy, and hence the reasons for patients coming to transplant are not always clear from registry data. Although this should be compensated by the use of factors such as age at transplant, disease phase, and time from diagnosis to transplant, some caution should be exercised in the interpretation of more recent results. Having said this, the analysis has been repeated recently for 3497 patients transplanted from 2007 to 2017 and confirmed improved outcome of 5-year OS across all-risk scores by 11-26\% (Fig. 76.2b). Although these pretransplant factors are known to affect outcome in all diseases, it is worth focusing specifically on the impact of disease phase in CML, in particular because one of the few problems of TKI therapy is that within the cohort of patients receiving transplants, the proportion transplanted in or after blast crisis has increased over time (Table 76.2).

Allografts for CML were initially restricted to patients in AP, and improvements in survival came only when transplant was performed in the CP. Data of 138 patients with CML transplanted between 1978 and 1982 and reported to the IBMTR showed 3-year survivals of $63 \%, 36 \%$, and $12 \%$ for patients transplanted in the $\mathrm{CP}, \mathrm{AP}$, and $\mathrm{BC}$, respectively. The probability of relapse for those transplanted in CP was 7\% (Speck et al. 1984). The effect of disease phase on the outcome of transplantation has not changed over the years. To optimize the effect of allo-HSCT for a
Modified from (Gratwohl et al. 1998). (b) OS curves supplied by Mrs. Linda Koster for the EBMT CMWP and based on 3497 patients transplanted from 2007 to 2017

Table 76.2 Change in proportion of patients transplanted in each disease phase from 2007 to 2017

\begin{tabular}{|l|l|l|l|l|}
$\begin{array}{l}\text { Year of } \\
\text { transplant }\end{array}$ & $\begin{array}{l}\text { 1st CP } \\
\text { Number } \\
\text { (\% total) }\end{array}$ & $\begin{array}{l}\text { AP } \\
\text { (\% total) }\end{array}$ & $\begin{array}{l}\geq 2 \text { CP } \\
\text { Number } \\
\text { (\% total) }\end{array}$ & $\begin{array}{l}\text { BC } \\
\text { Number } \\
(\% \text { total) }\end{array}$ \\
\hline 2007 & $164(50)$ & $49(15)$ & $82(25)$ & $34(10)$ \\
\hline 2008 & $134(45)$ & $34(12)$ & $84(28)$ & $44(15)$ \\
\hline 2009 & $133(41)$ & $46(14)$ & $92(28)$ & $53(16)$ \\
\hline 2010 & $128(36)$ & $57(16)$ & $106(30)$ & $65(18)$ \\
\hline 2011 & $148(49)$ & $50(15)$ & $86(26)$ & $46(14)$ \\
\hline 2012 & $127(46)$ & $34(12)$ & $74(27)$ & $42(15)$ \\
\hline 2013 & $136(44)$ & $41(13)$ & $78(25)$ & $54(18)$ \\
\hline 2014 & $138(43)$ & $48(15)$ & $75(24)$ & $57(18)$ \\
\hline 2015 & $137(44)$ & $43(14)$ & $73(23)$ & $62(20)$ \\
\hline 2016 & $111(42)$ & $30(11)$ & $70(26)$ & $55(21)$ \\
\hline 2017 & $74(36)$ & $20(10)$ & $68(33)$ & $45(22)$ \\
\hline
\end{tabular}

Data provided by Mrs. Linda Koster on behalf of the EBMT CMWP

patient who has progressed to blast crisis, a second CP should be achieved using TKI and/or conventional combination chemotherapy.

\subsubsection{Pretransplantation Treatment}

Early descriptions of therapy included radiotherapy, introduced at the beginning of the twentieth century and later oral chemotherapy, in particular BU and hydroxycarbamide. These approaches could control the signs and symptoms of CML in chronic phase but could not prevent its inevitable transformation into a rapidly fatal chemoresistant 
blastic disease. The first treatment that eradicated the Ph-positive clone and induced cure was BMT, initially described in syngeneic twins and soon followed by procedures involving HLA-matched siblings and later URD. Transplantation, once the treatment of choice for this disease, has been relegated to second-, third-, and even fourth-line treatment in parallel with the development of the TKI. As more potent TKI move to first-line therapy, patients destined to respond poorly to these drugs are identified earlier, and transplant will return to use as an earlier line strategy.

\subsubsection{Autologous HSCT}

Autologous HSCT for CML started about at the same time as allo-HSCT in the late 1970s early 1980s in Europe with the goal to set up the clock to early phase with high-dose therapy followed by reinfusion of autologous HSC. However, following the introduction of targeted therapy with TKI, the number of auto-HSCT in Europe has decreased rapidly, with only 0-4 per year between 2012 and 2016 as per the EBMT registry data. Auto-HSCT is currently not a recommended strategy in CML; however, it should be mentioned that due to the lack of randomized studies, the potential role of autologous HSCT for CML remains unknown.

\subsubsection{Allogeneic HSCT}

\subsubsection{Indication}

Although the introduction of TKI in the early 2000s dramatically changed the therapeutic strategy for CML, allo-HSCT has still a place, offering a very long-term PFS. This is particularly true as the leukemic quiescent stem cells are not dependent on BCR-ABL signaling for survival, and therefore those cells are not targeted by TKIs leading to a proportion of patients who will relapse or will have resistant disease despite TKI treatment. With extended follow-up, it appears that some $60 \%$ of patients can achieve excellent long-term disease control on imatinib, and a small proportion may even be able to stop treatment without experiencing disease recurrence. Approximately half of this group will achieve or regain remission on one of the second-generation TKI (2ndGTKI), bosutinib, dasatinib, and nilotinib, or third-generation TKI (3rdGTKI) ponatinib which is the only one that is effective against T315I mutation.

The efficacy of 2ndGTKI has led to their use as first-line therapy, and recently completed phase III studies suggest that approximately $80 \%$ of patients will achieve complete cytogenetic remissions within the first year, compared to $65 \%$ on imatinib. Based on these results, dasatinib and nilotinib have both been licensed for use in newly diagnosed patients. However, allo-HSCT remains the therapy of choice for advanced phase CML as well as for those with CP who failed to respond, develop TKIresistant mutations, and lose an established response and/or are intolerant of the drug.

The time to proceed to transplant remains controversial. This is particularly true for the substantial number of patients being started on 2ndGTKI as first-line therapy, who, in case of resistance, progression, or relapse, may be rescued with either another 2ndGTKI or 3rdGTKI, and then the question to proceed to transplant immediately or wait for another progression and third-line therapy rescue before to have allo-HSCT is a matter of debate. This is less true for those who are failing third-line therapy or have T315I mutation for whom allo-HSCT is recommended.

A number of national and international study groups are now reporting that long-term response to imatinib and 2ndGTKI can be predicted by the rate of fall of BCR-ABL transcript levels (as measured by RQ-PCR at 3 and 6 months). It is therefore possible to identify the patient destined for transplant within the first year of diagnosis while still in $\mathrm{CP}$ and return to a more measured approach to transplant. Recently the CMWP of the EBMT analyzed the data of patients transplanted for CML in the 3rdGTKI era that showed that the number of TKI given prior to allo-HSCT seems not to impact on the outcome; however, the stage of the disease as well as the performance status of patients did have an important impact (Chalandon et al. 2018). It is therefore very important to try to keep patients in first $\mathrm{CP}$ and avoid progression, even for those rescued to second or more $\mathrm{CP}$ after having progressed to 
advanced phase, as the results after transplantation are worse in this category. Allo-HSCT is also recommended for patients with $\mathrm{BC}$ after debulking with second or 3rdGTKI plus induction chemotherapy. For AP CML patients, this should be individualized, but the search for a donor and referral to a transplant center should be done rapidly, and transplant should be initiated after obtaining a new response to TKI for those progressing from CP to AP under therapy as their outcome is not good without allo-HSCT.

\subsubsection{Source of SC}

About two-thirds of the transplantation done nowadays for CML use PBSC as source of HSC (Chalandon et al. 2018); this is close to what is seen in other hematological malignancies (Holtick et al. 2014). It appears that there is no difference in general outcome depending on the stem cell source, although BM seems to have a decrease incidence on chronic GvHD and its severity. The source of stem is therefore left open, but PBSC may potentially be preferred to decrease the risk of graft failure and the relapse risk in more advanced disease, particularly with the use of RIC.

\subsubsection{Conditioning and GvHD Prophylaxis}

For CML patients, the best conditioning regimen as well as the best GvHD prophylaxis remains to be determined. Regarding the MAC, CY combined either with BU or TBI is still the one that has shown the best overall long-term survival (Copelan 2006). RIC that has been introduced later to offer transplantation to older patients or with more comorbidities did not show improved outcome over MAC, particularly in relation with a higher incidence of relapse with RIC (Kebriaei et al. 2007; Chalandon et al. 2018). Therefore, for elderly patients or those with comorbidities, RIC (FLU with BU or MEL) will be the choice, and for the others, particularly with advanced phases in order to control better the disease, MAC should be proposed.

For GvHD prophylaxis, CSA combined with short course MTX seems also to remain the standard for allo-HSCT for CML (Copelan 2006). In order to reduce the incidence and severity of GvHD, TCD was introduced in the 1980s; how- ever, there was an increase of relapse rate (Apperley et al. 1986). This led to many groups abandoning the use of TCD in sibling allografts for CML and often also in URD procedures. Others continued with its use and have reported good outcomes in sibling transplants, particularly following the introduction of DLI. In a small series of 23 CML patients with a median age of 36 years (range 18-58 years) transplanted with sibling donors and MAC between 1998 and 2016 at the University Hospital of Geneva using partial TCD with Campath-1H (alemtuzumab), the 15-year OS and LFS was 95\% using the strategy of escalating doses DLI for early molecular relapses with a low incidence of acute and chronic GvHD (Chalandon, unpublished data).

\subsubsection{Post transplant Strategies}

After allo-HSCT, rising or persistently high levels of BCR-ABL1 mRNA can be detected prior to cytogenetic or hematological relapse. Low or falling BCR-ABL1 transcript levels are associated with continuous remission, while high or rising transcript levels predict relapse. Therefore monitoring BCR-ABL1 post-allo-HSCT for CML is of utmost importance, even in the long term, due to relapses that have occurred up to more than 15 years post-HSCT.

Many CML patients will remain RQ-PCR positive during the first 3 months after alloHSCT, especially in the era of RIC or using TCD. In patients who are at least 4 months postallo-HSCT, one working definition of molecular relapse is one of the following:

(a) BCR-ABL/ABL1 ratio higher than $0.02 \%$ in three samples a minimum of 4 weeks apart.

(b) Clearly rising $\mathrm{BCR}-\mathrm{ABL} / \mathrm{ABL} 1$ ratio in three samples a minimum of 4 weeks apart with the last two higher than $0.02 \%$.

(c) BCR-ABL/ABL1 ratio higher than $0.05 \%$ in two samples a minimum of 4 weeks apart (Kaeda et al. 2006).

Administration of DLI can re-induce remission in $60-90 \%$ of patients with CML transplanted in, and relapsing in CP. The use of escalating doses in case of persistent disease reduces the risk of GvHD 
(Mackinnon et al. 1995). An EBMT study showed 69\% 5-year survival in 328 patients who received DLI for relapsed CML. DLI-related mortality was $11 \%$, and disease-related mortality was $20 \%$. Some form of GvHD was observed in $38 \%$ of patients. Risk factors for developing GvHD after DLI were T-cell dose at first DLI, time interval from transplant to DLI and donor type. In a timedependent multivariate analysis, GvHD after DLI was associated with a 2.3-fold increase in risk of death as compared with patients without GvHD (Chalandon et al. 2010).

With the advent of TKI, the CML post transplant interventions are more complexes but give more opportunities to rescue patients. It is possible to combine DLI and TKI for relapsing patients; however, the best order (TKI first, DLI first, or both combined) has not yet been defined. The CMWP of the EBMT reported 431 patients with CML relapses post-allo-HSCT who received TKI either alone $(55 \%)$ or in combination with DLI ( $14.5 \%$ before, $4.4 \%$ at the same time, and $26 \%$ after TKI). Only $42 \%$ of the patient obtained either a complete molecular (17.7\%), cytogenetic (4.4\%), or hematological $(20.2 \%)$ remission with a 5-year OS of $60 \%$ and of $47 \%$ for RFS (Chalandon et al. 2017). This rather low response rate may be in relation with the fact that 235 patients were transplanted for advanced phases (AP, BC or > CP1).

\section{Key Points}

- With the advent of targeted therapy, allo-HSCT use has decreased; however, it is of importance to monitor closely patients who are under TKI and avoid that they progress to advanced phase (AP or BC) as the outcome after transplant is better for CP1 as compared to all other conditions.

- Allo-HSCT should be considered for patient with $\mathrm{BC}$ after their return to $\mathrm{CP}$, for those progressing from $\mathrm{CP}$ to $\mathrm{AP}$ and for the one in $\mathrm{CP}$ after failure of thirdline therapy or with T315I mutation.

\section{References}

Apperley JF, Jones L, Hale G, et al. Bone marrow transplantation for patients with chronic myeloid leukaemia: T-cell depletion with Campath-1 reduces the incidence of graft-versus-host disease but may increase the risk of leukaemic relapse. Bone Marrow Transplant. 1986;1:53-66.

Byrne JL, Beshti H, Clark D, et al. Induction of remission after donor leucocyte infusion for the treatment of relapsed chronic idiopathic myelofibrosis following allogeneic transplantation: evidence for a 'graft vs. myelofibrosis' effect. Br J Haematol. 2000;108:430-3.

Cervantes F, Dupriez B, Pereira A, et al. New prognostic scoring system for primary myelofibrosis based on a study of the International Working Group for Myelofibrosis Research and Treatment. Blood. 2009;113:2895-901.

Chalandon Y, Passweg JR, Schmid C, et al. Outcome of patients developing GVHD after DLI given to treat CML relapse: a study by the Chronic Leukemia Working Party of the EBMT. Bone Marrow Transplant. 2010;45:558-64.

Chalandon Y, Iacobelli S, Hoek $\mathrm{J}$ et al. Use of first or second generation TKI for CML after allogeneic hematopoietic stem cell transplantation: a study by the CMWP of the EBMT. EBMT annual meeting, Marseille, France, 2017.

Chalandon Y, Sbianchi G, Hoek J et al. Allogeneic stem cell transplantation in patients with CML-CP in the era of third generation tyrosine kinase inhibitors: a study by the CMWP of the EBMT. EBMT annual meeting, Lisbon, Portugal, 2018.

Copelan EA. Hematopoietic stem-cell transplantation. N Engl J Med. 2006;354:1813-26.

Deeg HJ, Gooley TA, Flowers ME, et al. Allogeneic hematopoietic stem cell transplantation for myelofibrosis. Blood. 2003;102:3912-8.

Gangat N, Caramazza D, Vaidya R, et al. DIPSS plus: a refined Dynamic International Prognostic Scoring System for primary myelofibrosis that incorporates prognostic information from karyotype, platelet count, and transfusion status. J Clin Oncol. 2011;29:392-7.

Gratwohl A, Hermans J, Goldman JM, et al. Risk assessment for patients with chronic myeloid leukaemia before allogeneic blood or marrow transplantation. Chronic Leukemia Working Party of the European Group for Blood and Marrow Transplantation. Lancet. 1998;352:1087-92.

Guardiola P, Anderson JE, Bandini G, et al. Allogeneic stem cell transplantation for agnogenic myeloid metaplasia: a European Group for Blood and Marrow Transplantation, Societe Francaise de Greffe de Moelle, Gruppo Italiano per il Trapianto del Midollo Osseo, and Fred Hutchinson Cancer Research Center Collaborative Study. Blood. 1999;93:2831-8.

Guglielmelli P, Lasho TL, Rotunno G, et al. MIPSS70: mutation-enhanced International Prognostic Score 
System for transplantation-age patients with primary myelofibrosis. J Clin Oncol. 2018;36:310-8.

Hasford J, Pfirmann M, Hehlmann R, et al. A new prognostic score for survival of patients with chronic myeloid leukemia treated with interferon alfa. Writing Committee for the Collaborative CML Prognostic Factors Project Group. J Natl Cancer Inst. 1998;90:850-8.

Hoglund M, Sandin F, Simonsson B. Epidemiology of chronic myeloid leukaemia: an update. Ann Hematol. 2015;94(Suppl 2):S241-7.

Holtick U, Albrecht M, Chemnitz JM, et al. Bone marrow versus peripheral blood allogeneic haematopoietic stem cell transplantation for haematological malignancies in adults. Cochrane Database of Syst Rev. 2014;4:CD010189.

Kaeda J, O'Shea D, Szydlo RM, et al. Serial measurement of BCR-ABL transcripts in the peripheral blood after allogeneic stem cell transplantation for chronic myeloid leukemia: an attempt to define patients who may not require further therapy. Blood. 2006;107:4171-6.

Kebriaei P, Detry MA, Giralt S, et al. Long-term follow-up of allogeneic hematopoietic stem-cell transplantation with reduced-intensity conditioning for patients with chronic myeloid leukemia. Blood. 2007;110:3456-62.

Kroger N, Giorgino T, Scott BL, et al. Impact of allogeneic stem cell transplantation on survival of patients less than 65 years of age with primary myelofibrosis. Blood. 2015b;125:3347-50.

Kroger N, Holler E, Kobbe G, et al. Allogeneic stem cell transplantation after reduced-intensity conditioning in patients with myelofibrosis: a prospective, multicenter study of the Chronic Leukemia Working Party of the European Group for Blood and Marrow Transplantation. Blood. 2009;114:5264-70.

Kroger NM, Deeg JH, Olavarria E, et al. Indication and management of allogeneic stem cell transplantation in primary myelofibrosis: a consensus process by an EBMT/ELN international working group. Leukemia. 2015a;29:2126-33.

Lussana F, Rambaldi A, Finazzi MC, et al. Allogeneic hematopoietic stem cell transplantation in patients with polycythemia vera or essential thrombocythemia transformed to myelofibrosis or acute myeloid leukemia: a report from the MPN Subcommittee of the Chronic Malignancies Working Party of the European Group for Blood and Marrow Transplantation. Haematologica. 2014;99:916-21.

Mackinnon S, Papadopoulos EB, Carabasi MH, et al. Adoptive immunotherapy evaluating escalating doses of donor leukocytes for relapse of chronic myeloid leukemia after bone marrow transplantation: separa- tion of graft-versus-leukemia responses from graftversus-host disease. Blood. 1995;86:1261-8.

Passamonti F, Cervantes F, Vannucchi AM, et al. A dynamic prognostic model to predict survival in primary myelofibrosis: a study by the IWG-MRT (International Working Group for Myeloproliferative Neoplasms Research and Treatment). Blood. 2010;115:1703-8.

Passamonti F, Giorgino T, Mora B, et al. A clinicalmolecular prognostic model to predict survival in patients with post polycythemia vera and post essential thrombocythemia myelofibrosis. Leukemia. 2017;31:2726-31.

Raj K, Olavarria E, Eikema D-J, et al. Family mismatched donor transplantation for myelofibrosis: a retrospective analysis of the EBMT chronic leukaemia working party. Blood. 2016;128:4655.

Robin M, Giannotti F, Deconinck E, et al. Unrelated cord blood transplantation for patients with primary or secondary myelofibrosis. Biol Blood Marrow Transplant. 2014;20:1841-6.

Rondelli D, Goldberg JD, Isola L, et al. MPD-RC 101 prospective study of reduced-intensity allogeneic hematopoietic stem cell transplantation in patients with myelofibrosis. Blood. 2014;124:1183-91.

Scott BL, Gooley TA, Sorror ML, et al. The Dynamic International Prognostic Scoring System for myelofibrosis predicts outcomes after hematopoietic cell transplantation. Blood. 2012;119:2657-64.

Shanavas M, Popat U, Michaelis LC, et al. Outcomes of allogeneic hematopoietic cell transplantation in patients with myelofibrosis with prior exposure to Janus kinase 1/2 inhibitors. Biol Blood Marrow Transplant. 2016;22:432-40.

Sokal JE, Cox EB, Baccarani M, et al. Prognostic discrimination in "good-risk" chronic granulocytic leukemia. Blood. 1984;63:789-99.

Speck B, Bortin MM, Champlin R, et al. Allogeneic bonemarrow transplantation for chronic myelogenous leukaemia. Lancet. 1984;1:665-8.

Tefferi A, Mesa RA, Nagorney DM, Schroeder G, Silverstein MN. Splenectomy in myelofibrosis with myeloid metaplasia: a single-institution experience with 223 patients. Blood. 2000;95:2226-33.

Thiele J, Kvasnicka HM, Dietrich H, et al. Dynamics of bone marrow changes in patients with chronic idiopathic myelofibrosis following allogeneic stem cell transplantation. Histol Histopathol. 2005;20:879-89.

Wolschke C, Badbaran A, Zabelina $\mathrm{T}$, et al. Impact of molecular residual disease post allografting in myelofibrosis patients. Bone Marrow Transplant. 2017;52:1526-9. 
Open Access This chapter is licensed under the terms of the Creative Commons Attribution 4.0 International License (http://creativecommons.org/licenses/by/4.0/), which permits use, sharing, adaptation, distribution and reproduction in any medium or format, as long as you give appropriate credit to the original author(s) and the source, provide a link to the Creative Commons license and indicate if changes were made.

The images or other third party material in this chapter are included in the chapter's Creative Commons license, unless indicated otherwise in a credit line to the material. If material is not included in the chapter's Creative Commons license and your intended use is not permitted by statutory regulation or exceeds the permitted use, you will need to obtain permission directly from the copyright holder.

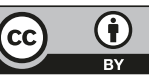

